\title{
FREE LIE ALGEBRAS AS MODULES OVER THEIR ENVELOPING ALGEBRAS
}

\author{
JOHN P. LABUTE
}

\begin{abstract}
In this paper we determine the linear relations that exist between the free generators of a free Lie algebra $L$ when it is viewed as a module over its enveloping algebra via the adjoint representation. As an application, the annihilator of a homogeneous element of $L$ is determined.
\end{abstract}

1. Statement of results. Let $K$ be a commutative associative ring with unit, let $L$ be a free Lie algebra over $K$ (cf. [1]), and let $U$ be the enveloping algebra of $L$. We identify $L$ with its image under the canonical injection of $L$ into $U$. We are interested in the structure of $L$ as a left $U$-module via the adjoint representation ad: $U \rightarrow \operatorname{End}_{K}(L)$.

Let $x_{1}, \ldots, x_{n}$ be arbitrary nonzero elements of $L$. Let $e_{1}, \ldots, e_{n}$ be the usual basis of the (left) $U$-module $U^{n}$ :

$$
e_{i}=\left(d_{i 1}, \ldots, d_{i n}\right)
$$

with $d_{i k}=1$ for $k=i$ and zero otherwise. For $1 \leqslant i, j \leqslant n, u, v \in U$, define $e_{i}(u), e_{i j}(u, v) \in U^{n}$ by

$$
\begin{aligned}
e_{i}(u) & =\left(\operatorname{ad}(u) x_{i}\right) u e_{i}, \\
e_{i j}(u, v) & =\left(\operatorname{ad}(v) x_{j}\right) u e_{i}+\left(\operatorname{ad}(u) x_{i}\right) v e_{j},
\end{aligned}
$$

and let $E$ be the $U$-submodule of $U^{n}$ generated by the elements $e_{i}(u), e_{i j}(u, v)$ with $1 \leqslant i, j \leqslant n, u, v \in U$. If $x_{1}, \ldots, x_{n}$ are homogeneous for some grading of the Lie algebra $L$, then the elements $u, v$ above can be taken to be homogeneous for the natural grading of $U$ induced by the grading of $L$. Hence, in this case, $E$ is a homogeneous submodule for the grading of $U^{n}$ defined by saying that $\left(u_{1}, \ldots, u_{n}\right) \in U^{n}$ is homogeneous of degree $k$ if and only if $u_{1}, \ldots, u_{n} \in U$ are homogeneous of degree $k$. If $\left(u_{1}, \ldots, u_{n}\right) \in E$, then we always have the relation

$$
\operatorname{ad}\left(u_{1}\right) x_{1}+\cdots+\operatorname{ad}\left(u_{n}\right) x_{n}=0 .
$$

THEOREM 1. If $x_{1}, \ldots, x_{n}$ is a free generating system for $L$, then

$$
\operatorname{ad}\left(u_{1}\right) x_{1}+\cdots+\operatorname{ad}\left(u_{n}\right) x_{n}=0 \quad \text { iff }\left(u_{1}, \ldots, u_{n}\right) \in E .
$$

Received by the editors November 3, 1976 and, in revised form, May 16, 1977. AMS (MOS) subject classifications (1970). Primary 17B35, 17B65; Secondary 16A06. Key words and phrases. Free Lie algebra, adjoint representation, annihilator. 
The proof of this theorem (for which we are indebted to the referee) is a minor adaptation of an argument in [2].

Now let $I$ be the ideal of $L$ generated by $x_{1}, \ldots, x_{n}$ and let $W$ be the enveloping algebra of $L / I$. Consider the following conditions on $x_{1}, \ldots, x_{n}$ :

(1) The elements $x_{1}, \ldots, x_{n}$ are homogeneous for some grading of $L$;

(2) The ideal I is a free Lie algebra;

(3) The quotient $L / I$ is a free $K$-module;

(4) The quotient $I /[I, I]$ is a free $W$-module of rank $n$ with basis $x_{1}, \ldots, x_{n}$ $(\bmod [I, I])$.

These conditions are satisfied if $x_{1}, \ldots, x_{n}$ is part of a free generating system for $L$ (cf. [1, §2, Proposition 10]) or if $n=1, K$ is a field, and $x_{1}$ is homogeneous and nonzero (cf. [3]).

THEOREM 2. If conditions (1), (2), (3), (4) hold, then the conclusion of Theorem 1 remains valid.

COROLlaRY 1. If $x_{1}, \ldots, x_{n}$ is part of a free generating system for $L$, then

$$
\operatorname{ad}\left(u_{1}\right) x_{1}+\cdots+\operatorname{ad}\left(u_{n}\right) x_{n}=0 \quad \text { iff }\left(u_{1}, \ldots, u_{n}\right) \in E .
$$

COROllary 2. If $x$ is a nonzero homogeneous element of $L$, and if $K$ is a field, then $\operatorname{ad}(u) x=0$ if and only if

$$
u=\sum_{v \in U} w_{v}(\operatorname{ad}(v) x) v \quad\left(w_{v} \in U\right)
$$

In [4] we use Corollary 2 in an essential way to determine the Lie algebra associated to the lower central series of the group $\left\langle x, y: x^{p}=1\right\rangle$ ( $p$ a prime). We do not know whether the homogeneity condition can be dropped in Corollary 2.

As was pointed out to us by the referee, Corollary 2 includes as a special case the known result (cf. [5, Theorem 5.10]) that two homogeneous elements of a free Lie algebra over a field $K$ commute if and only if they are linearly dependent. However, the result in [5] is more general since there it is not assumed that $K$ is a field.

2. Proof of Theorem 1. Complete $x_{1}, \ldots, x_{n}$ to a Hall basis $H$ of $L$ (cf. [1]). The elements of $H$ are homogeneous (for the natural grading of $L$ ), form an ordered set, and are defined inductively as follows:

(i) The elements $u \in H$ of degree $d(u)=1$ are $x_{1}, \ldots, x_{n}$.

(ii) The elements $u \in H$ of a given degree $\geqslant 1$ are ordered in an arbitrary manner. If $u, v \in H$, then $u<v$ if $d(u)<d(v)$.

(iii) If $u, v \in H$ with $u<v$, then $[u, v] \in H$ if $d(v)=1$ or if $v=\left[v_{1}, v_{2}\right]$ with $v_{1}, v_{2} \in H$ and $v_{2}>v_{1} \leqslant u$.

Every element of $H$ can be uniquely written in the form $\operatorname{ad}\left(u_{k} \ldots u_{1}\right) x_{j}$ where (a) $k \geqslant 0$; (b) $u_{i} \in H$ for $1 \leqslant i \leqslant k$; (c) $u_{1} \leqslant u_{2} \leqslant \cdots \leqslant u_{k}$; (d) $\operatorname{ad}\left(u_{k-i} \ldots u_{1}\right) x_{j}>u_{k-i+1}$ for $1 \leqslant i \leqslant k$. Conversely, such elements are elements of $H$. We call an element $u_{k} \ldots u_{1}$ normed of type $j$ if (a), (b), (c), (d) are satisfied. 
Let $N$ be the $K$-submodule of $U^{n}$ spanned by the elements of the form $w e_{i}$, where $w$ is normed of type $i$. The mapping $f: U^{n} \rightarrow L$ defined by

$$
f\left(u_{1}, \ldots, u_{n}\right)=\operatorname{ad}\left(u_{1}\right) x_{1}+\cdots+\operatorname{ad}\left(u_{n}\right) x_{n}
$$

is a $U$-module homomorphism of $U^{n}$ onto $L$ whose restriction to $N$ is bijective. To prove the theorem it suffices to show that $U^{n}=N+E$ since $E \subseteq \operatorname{Ker}(f)$. But this would follow if we could show that $v N \subseteq N+E$ for any $v \in H$. Let $v=\operatorname{ad}\left(v_{1} \ldots v_{l}\right) x_{i}$, where $v_{1} \ldots v_{l}$ is normed of type $i$, let $u_{1} \ldots u_{k}$ be normed of type $j$, and let $u=\operatorname{ad}\left(u_{1} \ldots u_{k}\right) x_{j}$. We want to show that $v u_{1} \ldots u_{k} e_{j} \in N+E$. Let $m=d(u)+d(v)$.

If $m=2$, we have $k=0$ and $v=x_{i}$. If $x_{i}=x_{j}$, we have $v e_{j} \in E$. If $x_{i}<x_{j}$, then $v$ is normed of type $j$ and $v e_{j} \in N$. If $x_{j}<x_{i}$, then

$$
v e_{j}=x_{i} e_{j} \equiv-x_{j} e_{i}(\bmod E)
$$

and, since $x_{j}$ is normed of type $i$, we have $v e_{j} \in N+E$. Hence the result holds if $m=2$.

We proceed by induction on $m$, assuming that $m>2$ and that the result holds for all pairs $\left(u^{\prime}, v^{\prime}\right)$ with $d\left(u^{\prime}\right)+d\left(v^{\prime}\right)<m$. We have

$$
\begin{aligned}
v u_{1} \ldots u_{k} e_{j} & =\left(\operatorname{ad}\left(v_{1} \ldots v_{l}\right) x_{i}\right) u_{1} \ldots u_{k} e_{j} \\
& \equiv-\left(\operatorname{ad}\left(u_{1} \ldots u_{k}\right) x_{j}\right) v_{1} \ldots v_{l} e_{i} \quad(\bmod E) \\
& =-u v_{1} \ldots v_{l} e_{i} .
\end{aligned}
$$

Also $u u_{1} \ldots u_{k} e_{j}=\left(\operatorname{ad}\left(u_{1} \ldots u_{k}\right) x_{j}\right) u_{1} \ldots u_{k} e_{j} \in E$. Hence, without loss of generality, we may assume that $v<u$. Hence $\min (d(u), d(v))=d(v)$. As $m>2$ we have $d(u)>1$, and so $k>0$.

(a) If $v \geqslant u_{1}$, then (as $v<u$ ) $v u_{1} \ldots u_{k}$ is normed of type $j$ and so $v u_{1} \ldots u_{k} e_{j} \in N$.

(b) If $\min (d(u), d(v))>m / 3$, then $d(u)=m-d(v)<2 m / 3$. As $u_{1} \ldots u_{k}$ is normed of type $j$ we have $u_{1}<\operatorname{ad}\left(u_{2} \ldots u_{k}\right) x_{j}$ and so $d\left(u_{1}\right) \leqslant$ $d(u) / 2<m / 3$. Thus $d\left(u_{1}\right)<d(v)$ and hence $u_{1}<v$. Then by (a) the result holds.

(c) Proceeding by downward induction on $\min (u, v)$, we assume that the result holds for all pairs $(w, z)$ with $z \in H, w=\operatorname{ad}\left(w_{1} \ldots w_{r}\right) x_{s}, w_{1} \ldots w_{r}$ normed of type $s, d(w)+d(z)=m$, and $\min (w, z)>\min (u, v)$. In view of (a), we may assume $u_{1}>v$. Now

$$
\begin{aligned}
v u_{1} \ldots u_{k} e_{j} & =\left(\operatorname{ad}\left(v_{1} \ldots v_{l}\right) x_{i}\right) u_{1} \ldots u_{k} e_{j} \\
& \equiv-\left(\operatorname{ad}\left(u_{1} \ldots u_{k}\right) x_{j}\right) v_{1} \ldots v_{l} e_{i} \quad(\bmod E) \\
& =-u_{1}\left\{\left(\operatorname{ad}\left(u_{2} \ldots u_{k}\right) x_{j}\right) v_{1} \ldots v_{l} e_{i}\right\} \\
& \quad+\left(\operatorname{ad}\left(u_{2} \ldots u_{k}\right) x_{j}\right)\left\{u_{1} v_{1} \ldots v_{l} e_{i}\right\} .
\end{aligned}
$$

The expressions in braces both have degree $<m$. Thus by the induction assumption they are congruent modulo $E$ to elements of $N$ of the same degree. Now $u_{1}>v, \operatorname{ad}\left(u_{2} \ldots u_{k}\right) x_{j}>u_{1}>v, d\left(\left(\operatorname{ad}\left(u_{2} \ldots u_{k}\right) x_{j}\right) v_{1} \ldots v_{l}\right)$ $\geqslant d(v)$, and $d\left(u_{1} v_{1} \ldots v_{l}\right) \geqslant d(v)$. Consequently $v u_{1} \ldots u_{k} e_{j}$ is a linear 
combination of terms of the form $z w_{1} \ldots w_{r} e_{s}$ with $z \in H, w_{1} \ldots w_{r}$ normed of type $s, z>v$, and $w=\operatorname{ad}\left(w_{1} \ldots w_{r}\right) x_{s}>v$. By induction, the result follows.

3. Proof of Theorem 2. Since $I$ is a homogeneous ideal of $L$ with $L / I$ a free $K$-module, we have $L=I+J$ with $J$ a free homogeneous $K$-submodule of $L$. Let $\left(t_{i}\right)$ be an ordered, homogeneous, $K$-module basis of $J$, and let $B$ be the set of elements of $U$ of the form

$$
t_{i_{1}} t_{i_{2}} \ldots t_{i_{k}} \quad\left(i_{1} \leqslant i_{2} \leqslant \cdots \leqslant i_{k}, k \geqslant 0\right) \text {. }
$$

Then, because of homogeneity, $I$ is a free Lie algebra over $K$ with free generating system $\left(\operatorname{ad}(w) x_{j}\right)_{w \in B, 1 \leqslant j \leqslant n}$. Moreover, every element $u \in U$ can be uniquely written in the form

$$
u=\sum_{w \in B} v_{w} w
$$

where $v_{w} \in V$, the enveloping algebra of $I$. Now suppose that

$$
\operatorname{ad}\left(u_{1}\right) x_{1}+\cdots+\operatorname{ad}\left(u_{n}\right) x_{n}=0
$$

and write

$$
u_{i}=\sum_{j=1}^{m} v_{i j} w_{j} \quad(1 \leqslant i \leqslant n)
$$

with $v_{i j} \in V$ and $w_{1}, \ldots, w_{m}$ distinct elements of $B$. Then, if $z_{i j}=\operatorname{ad}\left(w_{j}\right) x_{i}$, we have

$$
\sum_{i, j} \operatorname{ad}\left(v_{i j}\right) z_{i j}=0
$$

Moreover, by introducing zero elements $v_{i j}$ and increasing $m$, we can assume that the elements $v_{i j}$ are in the subalgebra $V^{\prime}$ of $V$ generated by the elements $z_{i j}$. Applying Theorem 1, we obtain that the family $\left(v_{i j}\right)$ is a $V^{\prime}$-linear combination of elements of the form

$$
\left(\operatorname{ad}(v) z_{i j}\right) v e_{i j}, \quad\left(\operatorname{ad}(v) z_{i j}\right) w e_{k l}+\left(\operatorname{ad}(w) z_{k l}\right) v e_{i j},
$$

where $v, w \in V^{\prime}$ and $e_{p q}$ is the family $\left(d_{i j}\right)$ with $d_{i j}=1$ if $p=i, q=j$ and zero otherwise. Since

$$
\left(u_{1}, \ldots, u_{n}\right)=\sum_{j=1}^{m}\left(v_{1 j}, \ldots, v_{n j}\right) w_{j},
$$

it follows that $\left(u_{1}, \ldots, u_{n}\right)$ is a $V^{\prime}$-linear combination of elements of the form

$$
\begin{gathered}
\left(\operatorname{ad}(v) z_{i j}\right) v w_{j} e_{i}=\left(\operatorname{ad}\left(v w_{j}\right) x_{i}\right) v w_{j} e_{i}, \\
\left(\operatorname{ad}(v) z_{i j}\right) w w_{l} e_{k}+\left(\operatorname{ad}(w) z_{k l}\right) v w_{j} e_{i} \\
=\left(\operatorname{ad}\left(v w_{j}\right) x_{i}\right) w w_{l} e_{k}+\left(\operatorname{ad}\left(w w_{l}\right) x_{k}\right) v w_{j} e_{i}
\end{gathered}
$$

which lie in $E$. Q.E.D. 


\section{REFERENCES}

1. N. Bourbaki, Groupes et algèbres de Lie, Ėléments de Mathématiques, Hermann, Paris, 1972, Chapitre 2.

2. Marshall Hall, Jr., A basis for free Lie rings and higher commutators in free groups, Proc. Amer. Math. Soc. 1 (1950), 575-581.

3. John P. Labute, Algebres de Lie et pro-p-groupes définis par une seule relation, Invent. Math. 4 (167), 142-158. MR 36 \# 1581.

4. The lower central series of the group $\left\langle x, y: x^{p}=1\right\rangle$ (to appear).

5. W. Magnus, A. Karrass and D. Solitar, Combinatorial group theory, Interscience, New York, 1966.

.Department of Mathematics, McGill University, Montreal, Quebec, Canada 